\title{
A NOTE ON QUASI LAURENT POLYNOMIAL ALGEBRAS IN $n$ VARIABLES
}

\author{
ASAWARI M. ABHYANKAR AND S.M. BHATWADEKAR
}

ABSTRACT. Let $S$ be a principal ideal domain. Recall that a Laurent polynomial algebra over $S$ is an $S$ algebra of the form $S\left[T_{1}, \ldots, T_{n}, T_{1}^{-1}, \ldots, T_{n}^{-1}\right]$. Generalizing this notion, we call an $S$-algebra of the form $S\left[T_{1}, \ldots, T_{n}, f_{1}^{-1}, \ldots, f_{n}^{-1}\right]$ a quasi Laurent polynomial algebra in $n$ variables over $S$ if $T_{1}, \ldots, T_{n}$ are algebraically independent over $S$ and $f_{i}=a_{i} T_{i}+b_{i}$, where $a_{i} \in S \backslash 0$ and $b_{i} \in S$ are such that $\left(a_{i}, b_{i}\right) S=S$, for each $i=1, \ldots, n$. It has been shown recently that a locally Laurent polynomial algebra in $n$ variables over $S$ is itself a Laurent polynomial algebra. Now suppose $A$ is a locally quasi Laurent polynomial algebra in $n$ variables over $S$. In this note, we investigate the question: 'is $A$ necessarily quasi Laurent polynomial in $n$ variables over $S$ ?' We first give a sufficient condition for the question to have an affirmative answer. Moreover, when $S$ is semi-local with two maximal ideals and contains the field of rationals $\mathbf{Q}$, we give examples of $S$-algebras which are locally quasi Laurent polynomial in two variables but not quasi Laurent polynomial in two variables.

1. Introduction. In [1] , the following notion of a quasi $\mathbf{A}^{*}$ algebra over an integral domain $S$ has been introduced: an $S$-algebra $C$ is said to be quasi $\mathbf{A}^{*}$ if there exists an element $T$ in $C$ which is transcendental over $S$ such that

$$
C=S\left[T,(a T+b)^{-1}\right]
$$

for some $a \in S \backslash 0, b \in S$ satisfying $(a, b) S=S$.

In a similar manner (keeping in mind that an $S$-algebra of the form $S\left[T, T^{-1}\right]$ is referred to as " $A^{*}$ " over $S$ ), we call an $S$-algebra $C$ "quasi Laurent polynomial (quasi LP) in $n$ variables over $S$ " if there exist elements $T_{1}, \ldots, T_{n}$ in $C$ which are algebraically independent over

2010 AMS Mathematics subject classification. Primary 16S34, Secondary 13F10, $13 \mathrm{~F} 20$.

Keywords and phrases. Polynomial algebra, Laurent polynomial algebra, quasi Laurent polynomial algebra, principal ideal domain.

Received by the editors on January 7, 2013. 
$S$ such that

$$
C=S\left[T_{1}, \ldots, T_{n},\left(a_{1} T_{1}+b_{1}\right)^{-1}, \ldots,\left(a_{n} T_{n}+b_{n}\right)^{-1}\right]
$$

for some $a_{i} \in S \backslash 0, b_{i} \in S$ satisfying $\left(a_{i}, b_{i}\right) S=S$, for $i=1, \ldots, n$.

Let $C=S\left[T_{1}, \ldots, T_{n}, f_{1}^{-1}, \ldots, f_{n}^{-1}\right]$ be a quasi Laurent polynomial algebra in $n$ variables over $S$, where $f_{i}=a_{i} T_{i}+b_{i}$ for $i=1, \ldots, n$. Let $K$ be the quotient field of $S$. Then observe that:

(1) $C$ is faithfully flat over $S$,

(2) $C \otimes_{S} K$ is a Laurent polynomial (LP) algebra in $n$ variables over $K$, and if $a_{1}, \ldots, a_{n}$ are units in $S$, then $C$ is a Laurent polynomial algebra in $n$ variables over $S$.

We call an $S$-algebra $C$ locally quasi Laurent polynomial in $n$ variables over $S$ if $C \otimes_{S} S_{\mathfrak{m}}$ is quasi Laurent polynomial in $n$ variables over the local ring $S_{\mathfrak{m}}$ for every maximal ideal $\mathfrak{m}$ of $S$.

In [1, Corollary 4.5], it is proved that, if $S$ is a Noetherian factorial domain and $C$ is a finitely generated, faithfully flat $S$-algebra such that $C \otimes_{S} S_{P}$ is quasi $\mathbf{A}^{*}$ over $S_{P}$ for every height one prime ideal $P$ in $S$, then $C$ is quasi $\mathbf{A}^{*}$ over $S$. In particular, if $S$ is a principal ideal domain (P.I.D.) and $C$ is a finitely generated, locally quasi Laurent polynomial algebra in one variable over $S$, then $C$ is a quasi Laurent polynomial algebra in one variable. Moreover, a special case of Theorem 2.3 in [2] says that a locally Laurent polynomial algebra in $n$ variables over a P.I.D. $S$ is in fact a Laurent polynomial algebra over $S$.

In view of these results, it is natural to ask: let $S$ be a P.I.D. and $C$ a finitely generated S-algebra which is locally quasi LP in $n(\geq 2)$ variables. Is $C$ necessarily quasi $L P$ in $n$ variables over $S$ ?

In this note, we first give a sufficient condition for the above question to have an affirmative answer (Proposition 3.3). Subsequently, we show that the above question may not always have an affirmative answer implying that, in general, we cannot expect nice behavior in the case of a locally quasi Laurent polynomial algebra in $n(\geq 2)$ variables which is not a locally Laurent polynomial algebra (Examples 4.1, 4.5 and 4.6).

2. Preliminaries. All the rings in this note are assumed to be commutative and contain unity. For a ring $S$, let $S^{[n]}$ denote a polynomial ring in $n$ variables over $S$ and $S^{*}$ the multiplicative group 
of units in $S$. For a prime ideal $P$ of $S$ and an $S$-algebra $A, A_{P}$ denotes the ring $A \otimes_{S} S_{P}\left(=T^{-1} A\right)$, where $T=S \backslash P$.

We state below some results which can be proved easily.

Lemma 2.1. Let $S$ be an integral domain. Let $t_{1}, \ldots, t_{n}$ be non-zero elements of $S$. If $\pi$ is a prime element of $S$ such that $\pi \nmid t_{i}$ for each $i=1, \ldots, n$, then $\pi$ remains prime in $S\left[t_{1}^{-1}, \ldots, t_{n}^{-1}\right]$.

Lemma 2.2. Let $S$ be an integral domain. Let $\pi_{1}, \ldots, \pi_{n}$ be prime elements of $S$, no two of which are associates. Then each unit in $S\left[\pi_{1}^{-1}, \ldots, \pi_{n}^{-1}\right]$ is of the form $\lambda \pi_{1}^{r_{1}} \cdots \pi_{n}^{r_{n}}$ for some $\lambda \in S^{*}$ and integers $r_{1}, \ldots, r_{n}$.

Lemma 2.3. Let $S$ be an integral domain and $T$ an indeterminate over $S$. If $a \in S \backslash 0$ and $b \in S$ are such that $(a, b) S=S$, then $a T+b$ is $a$ prime element of $S[T]$.

Lemma 2.4. Let $S$ be an integral domain, $\pi$ a prime element of $S$ and $D=S[T, W]\left(=S^{[2]}\right)$. Let $h_{1} \in S[T], h_{2} \in S[W]$ and $\overline{h_{1}}, \overline{h_{2}}$ denote the images of $h_{1}$ and $h_{2}$ respectively, in $D / \pi D$. If $\overline{h_{1}}, \overline{h_{2}} \notin S / \pi S$, then $\overline{h_{1}}, \overline{h_{2}}$ are algebraically independent over $S / \pi S$.

Lemma 2.5. Let $D \subseteq B$ be integral domains. Suppose there exists a non-zero element $\pi$ in $D$ such that $D[1 / \pi]=B[1 / \pi]$ and the canonical map $D / \pi D \rightarrow B / \pi B$ is injective. Then $D=B$.

The following lemma will be required very often in Section 4.

Lemma 2.6. Let $S \subseteq B$ be integral domains. Let $X, Y$ be prime elements of $B$ which are algebraically independent over $S$ and $A=$ $B\left[X^{-1}, Y^{-1}\right]$. Suppose

(I) $B^{*}=S^{*}$ and

(II) $A=S\left[T, W, f^{-1}, g^{-1}\right]$, a quasi Laurent polynomial algebra in two variables over $S$, where $f=a T+b$ and $g=c W+d$.

Then, there exist $m, n, r, s, m^{\prime}, n^{\prime}, r^{\prime}, s^{\prime} \in \mathbf{Z}$ and $\lambda, \mu, \lambda_{1}, \mu_{1} \in S^{*}$ such that 
(i) $m s-n r=m^{\prime} s^{\prime}-n^{\prime} r^{\prime}= \pm 1$,

(ii) $X=\lambda f^{m} g^{n}, Y=\mu f^{r} g^{s}, f=\lambda_{1} X^{m^{\prime}} Y^{n^{\prime}}$ and $g=\mu_{1} X^{r^{\prime}} Y^{s^{\prime}}$.

In particular, $S\left[X, Y, X^{-1}, Y^{-1}\right]=S\left[f, g, f^{-1}, g^{-1}\right]$.

Proof. By the definition of a quasi Laurent polynomial algebra in two variables, we have $(a, b) S=(c, d) S=S$. Then, by Lemma 2.3, $f$ and $g$ are prime elements of $S[T, W]$. Hence, by Lemma 2.2, there exist $\lambda, \mu \in S^{*}$ and $m, n, r, s \in \mathbf{Z}$ such that

$$
X=\lambda f^{m} g^{n} \quad \text { and } \quad Y=\mu f^{r} g^{s} .
$$

On the other hand, by (I) and Lemma 2.2, the units $f, g$ of $A$ can be expressed as

$$
f=\lambda_{1} X^{m^{\prime}} Y^{n^{\prime}} \text { and } g=\mu_{1} X^{r^{\prime}} Y^{s^{\prime}}
$$

for some $\lambda_{1}, \mu_{1} \in S^{*}$ and $m^{\prime}, n^{\prime}, r^{\prime}, s^{\prime} \in \mathbf{Z}$. From (2.1) and (2.2), we obtain

$$
X=\left(\lambda \lambda_{1}^{m} \mu_{1}^{n}\right) X^{m m^{\prime}+n r^{\prime}} Y^{m n^{\prime}+n s^{\prime}}
$$

and

$$
Y=\left(\mu \lambda_{1}^{r} \mu_{1}^{s}\right) X^{r m^{\prime}+s r^{\prime}} Y^{r n^{\prime}+s s^{\prime}}
$$

Therefore,

$$
\left(\begin{array}{cc}
m & n \\
r & s
\end{array}\right)\left(\begin{array}{cc}
m^{\prime} & n^{\prime} \\
r^{\prime} & s^{\prime}
\end{array}\right)=\left(\begin{array}{ll}
1 & 0 \\
0 & 1
\end{array}\right)
$$

and hence $m, n, r, s, m^{\prime}, n^{\prime}, r^{\prime}, s^{\prime} \in \mathbf{Z}$ are such that $m s-n r=m^{\prime} s^{\prime}-$ $n^{\prime} r^{\prime}= \pm 1$.

Also, from (2.1) and (2.2), it follows that

$$
S\left[X, Y, X^{-1}, Y^{-1}\right]=S\left[f, g, f^{-1}, g^{-1}\right] .
$$

Lemma 2.7. Let $S$ be an integral domain. Let $A$ be a quasi Laurent polynomial algebra in $n$ variables over $S$. Then every prime element of $S$ remains prime in $A$.

Proof. Let $A=S\left[X_{1}, \ldots, X_{n}, f_{1}^{-1}, \ldots, f_{n}^{-1}\right]$, where $f_{i}=a_{i} X_{i}+b_{i}$ for $i=1, \ldots, n$. Let $\pi$ be a prime element of $S$. First, note that 
$\pi$ remains prime in $S\left[X_{1}, \ldots, X_{n}\right]$. Since $\left(a_{i}, b_{i}\right) S=S, \pi \nmid f_{i}$ in $S\left[X_{1}, \ldots, X_{n}\right]$ for each $i=1, \ldots, n$. Therefore, $\pi$ is a prime element of $A$ by Lemma 2.1 .

The following lemma is easy to prove.

Lemma 2.8. Let $S$ be an integral domain and $P=\pi S$ a prime ideal of $S$. Let $T \subseteq(S \backslash P)$ be a multiplicative subset of $S$. Then $S=S[1 / \pi] \cap T^{-1} S$ and $S^{*}=(S[1 / \pi])^{*} \cap\left(T^{-1} S\right)^{*}$. Moreover, if $A$ is a flat $S$-algebra, then $A=A[1 / \pi] \cap T^{-1} A$ and $A^{*}=(A[1 / \pi])^{*} \cap\left(T^{-1} A\right)^{*}$.

Lemma 2.9. Let $S$ be an integral domain and $P$ a prime ideal of $S$. Let $A$ be a flat $S$-algebra. If $P A_{P}$ is a prime ideal of $A_{P}$, then $P A$ is a prime ideal of $A$.

Proof. Since $A$ is $S$-flat,

$$
\begin{aligned}
S / P \hookrightarrow S_{P} / P S_{P} & \Longrightarrow A \otimes_{S} S / P \hookrightarrow A \otimes_{S} S_{P} / P S_{P} \\
& \Longrightarrow A / P A \hookrightarrow A_{P} / P A_{P} .
\end{aligned}
$$

Hence, the proof.

3. A sufficient condition. Let $S$ be a P.I.D. Let $A$ be an $S$-algebra. If $A$ is a locally Laurent polynomial algebra in $n$ variables over $S$, then by [2, Theorem 2.3], $A$ is a Laurent polynomial algebra over $S$. But, even if $n=1$, a locally quasi LP algebra is not necessarily quasi LP over $S$. In fact, by an example that follows, a locally quasi LP algebra in one variable over $S$ need not be even finitely generated over $S$.

Example 3.1. Let $S=\mathbf{Z}$ and $B=\mathbf{Z}[X, X / 2, X / 3, \ldots, X / p, \ldots]$ where $p$ varies over the set of prime integers. Let $f=X-1$ and $A=B\left[f^{-1}\right]$. For a prime integer $p$, let $P=p \mathbf{Z}$. Then $B_{P}=\mathbf{Z}_{P}[X / p]$, and hence $A$ is locally quasi Laurent polynomial in one variable over $\mathbf{Z}$. But, since $B$ is not finitely generated over $\mathbf{Z}, A$ cannot be a finitely generated Z-algebra, by [1, Theorem 5.7].

Now, let $A$ be a locally quasi Laurent polynomial algebra in $n$ variables over $S$ which also satisfies the following condition: 
$A[1 / \pi]$ is a Laurent polynomial algebra in $n$ variables over $S[1 / \pi]$ for some prime $\pi$ in $S$.

Observe that, under this condition, $A_{P}$ is a Laurent polynomial algebra in $n$ variables over $S_{P}$ for every maximal ideal $P(\neq \pi S)$ of $S$. In other words, we can say that $A$ is almost locally Laurent polynomial algebra in $n$ variables over $S$.

We show that this is a sufficient condition for $A$ to be quasi Laurent polynomial in $n$ variables over $S$. First we prove a lemma.

Lemma 3.2. Let $S$ be a factorial domain and $A$ a flat $S$-algebra. Let $\mathfrak{m}=\pi S$ be a prime ideal of $S$. Suppose

(1) $A_{\mathfrak{m}}=S_{\mathfrak{m}}\left[X_{1}, \ldots, X_{n}, f_{1}^{-1}, \ldots, f_{n}^{-1}\right]$, a quasi Laurent polynomial algebra in $n$ variables over $S_{\mathfrak{m}}$, where $f_{i}=a_{i} X_{i}+b_{i}$, for $i=1, \ldots, n$ and

(2) $A[1 / \pi]=S[1 / \pi]\left[U_{1}, \ldots, U_{n}, g_{1}^{-1}, \ldots, g_{n}^{-1}\right]$, a quasi Laurent polynomial algebra in $n$ variables over $S[1 / \pi]$, where $g_{i}=$ $c_{i} U_{i}+d_{i}$ for $i=1, \ldots, n$.

Then, $f_{1}, \ldots, f_{n}$ and $g_{1}, \ldots, g_{n}$ can be chosen such that:

$$
S\left[f_{1}, \ldots, f_{n}, f_{1}^{-1}, \ldots, f_{n}^{-1}\right]=S\left[g_{1}, \ldots, g_{n}, g_{1}^{-1}, \ldots, g_{n}^{-1}\right](\subseteq A) .
$$

Proof. From (1) and Lemma 2.7, we see that $\mathfrak{m} A_{\mathfrak{m}}$ is a prime ideal of $A_{\mathfrak{m}}$ and, since $A$ is $S$-flat, by Lemma $2.9, \pi$ remains prime in $A$. Now, let $Q=t S$ be a prime ideal of $S$, other than $\pi S$. From (2), it follows that $A_{Q}$ is a quasi Laurent polynomial algebra in $n$ variables over $S_{Q}$. Again, by Lemmas 2.7 and $2.9, t$ is a prime element of $A$. Thus, every prime element of $S$ remains prime in $A$.

Since $g_{1}, \ldots, g_{n}$ are units in $A[1 / \pi]$ and $\pi$ is a prime in $A$, by Lemma 2.2, we can write $g_{i}=\lambda_{i} \pi^{r_{i}}$ for some $\lambda_{i} \in A^{*}$ and $r_{i} \in \mathbf{Z}$ (for $i=1, \ldots, n$ ). Therefore, replacing $g_{i}$ by $\pi^{-r_{i}} g_{i}$, if required, we can assume that $g_{1}, \ldots, g_{n} \in A^{*}$.

Since $f_{1}, f_{1}^{-1} \in A_{\mathfrak{m}}$, write $f_{1}=a / s$ and $f_{1}^{-1}=a^{\prime} / s^{\prime}$, where $a, a^{\prime} \in A$ and $s, s^{\prime} \in S \backslash \mathfrak{m}$. Let $\left\{\pi_{1}, \ldots, \pi_{r}\right\}$ be the set of all distinct prime divisors of a least common multiple of $s$ and $s^{\prime}$ in $S$. Also assume that no two of $\pi_{1}, \ldots, \pi_{r}$ are associates. Then note that $f_{1}, f_{1}^{-1} \in A\left[\pi_{1}^{-1}, \ldots, \pi_{r}^{-1}\right]\left(\subseteq A_{\mathfrak{m}}\right)$, i.e., $f_{1}$ is a unit in $A\left[\pi_{1}^{-1}, \ldots, \pi_{r}^{-1}\right]$. By Lemma 2.2, we can write $f_{1}=\mu \pi_{1}^{l_{1}} \cdots \pi_{r}^{l_{r}}$, for some $\mu \in A^{*}$ and 
$l_{1}, \ldots, l_{r} \in \mathbf{Z}$. Replacing $f_{1}$ by $\mu$, if required, we can assume that $f_{1} \in A^{*}$. Similarly, assume that $f_{2}, \ldots, f_{n} \in A^{*}$.

From the above discussion, it follows that $f_{1}, \ldots, f_{n}$ and $g_{1}, \ldots, g_{n}$ can be chosen such that

$$
S\left[g_{1}, \ldots, g_{n}, g_{1}^{-1}, \ldots, g_{n}^{-1}\right] \subseteq A
$$

and

$$
S\left[f_{1}, \ldots, f_{n}, f_{1}^{-1}, \ldots, f_{n}^{-1}\right] \subseteq A .
$$

By Lemma $2.3, f_{1}, \ldots, f_{n}$ are prime elements of $S_{\mathfrak{m}}\left[X_{1}, \ldots, X_{n}\right]$. Therefore, by Lemma 2.2, for each $i(1 \leq i \leq n), g_{i} \in A^{*}\left(\subseteq A_{\mathfrak{m}}^{*}\right)$ can be expressed as

$$
g_{i}=\alpha_{i} f_{1}^{p_{i 1}} f_{2}^{p_{i 2}} \cdots f_{n}^{p_{i n}}
$$

for some $\alpha_{i} \in S_{\mathfrak{m}}^{*}$ and $p_{i j} \in \mathbf{Z}, 1 \leq j \leq n$.

Since $g_{1}, \ldots, g_{n}$ are prime elements of $S[1 / \pi]\left[U_{1}, \ldots, U_{n}\right]$, again using Lemma 2.2, for each $i(1 \leq i \leq n), f_{i} \in A^{*}\left(\subseteq(A[1 / \pi])^{*}\right)$ can be expressed as

$$
f_{i}=\beta_{i} g_{1}^{q_{i 1}} g_{2}^{q_{i 2}} \cdots g_{n}^{q_{i n}}
$$

for some $\beta_{i} \in(S[1 / \pi])^{*}$ and $q_{i j} \in \mathbf{Z}, 1 \leq j \leq n$.

Let $K$ be the quotient field of $S$. From equations (3.1) and (3.2), we obtain the following expressions (in $A \otimes_{S} K$ ):

$$
\begin{aligned}
& g_{i}=\alpha_{i} \beta_{1}^{p_{i 1}} \beta_{2}^{p_{i 2}} \cdots \beta_{n}^{p_{i n}} g_{1}^{p_{1}} g_{2}^{p_{2}} \cdots g_{n}^{p_{n}}, \\
& f_{i}=\beta_{i} \alpha_{1}^{q_{i 1}} \alpha_{2}^{q_{i 2}} \cdots \alpha_{n}^{q_{i n}} f_{1}^{q_{1}} f_{2}^{q_{2}} \cdots f_{n}^{q_{n}},
\end{aligned}
$$

where $p_{r}=\sum_{j=1}^{n} p_{i j} q_{j r}$ and $q_{r}=\sum_{j=1}^{n} q_{i j} p_{j r}$ for $r=1, \ldots, n$.

From the above expressions, it follows that (in $K$ ),

$$
\alpha_{i} \beta_{1}^{p_{i 1}} \beta_{2}^{p_{i 2}} \cdots \beta_{n}^{p_{i n}}=1 \Longrightarrow \alpha_{i}=\beta_{1}^{-p_{i 1}} \beta_{2}^{-p_{i 2}} \cdots \beta_{n}^{-p_{i n}}
$$

and

$$
\beta_{i} \alpha_{1}^{q_{i 1}} \alpha_{2}^{q_{i 2}} \cdots \alpha_{n}^{q_{i n}}=1 \Longrightarrow \beta_{i}=\alpha_{1}^{-q_{i 1}} \alpha_{2}^{-q_{i 2}} \cdots \alpha_{n}^{-q_{i n}} .
$$


Hence $\alpha_{i}, \beta_{i} \in S_{\mathfrak{m}}^{*} \cap(S[1 / \pi])^{*}=S^{*}$ (by Lemma 2.8). As a consequence of this, from equations (3.1) and (3.2), we see that

$$
S\left[g_{1}, \ldots, g_{n}, g_{1}^{-1}, \ldots, g_{n}^{-1}\right]=S\left[f_{1}, \ldots, f_{n}, f_{1}^{-1}, \ldots, f_{n}^{-1}\right] .
$$

Now we prove the following proposition.

Proposition 3.3. Let $S$ be a factorial domain and $A$ a faithfully flat S-algebra. Suppose

(1) $A_{P}$ is quasi Laurent polynomial in $n$ variables over $S_{P}$ for every height one prime ideal $P$ of $S$ and

(2) $A[1 / \pi]$ is a Laurent polynomial algebra in $n$ variables over $S[1 / \pi]$ for some prime element $\pi$ in $S$.

Then $A$ is quasi Laurent polynomial in $n$ variables over $S$. As a consequence, $A$ is finitely generated over $S$.

Proof. From (1), Lemma 2.7 and Lemma 2.9, it follows that every prime element of $S$ remains prime in $A$. Let $\mathfrak{m}=\pi S$. Since $A[1 / \pi]$ is a Laurent polynomial algebra in $n$ variables over $S[1 / \pi]$, there exist $U_{1}, \ldots, U_{n} \in A[1 / \pi]$ which are algebraically independent over $S[1 / \pi]$ such that

$$
A[1 / \pi]=S[1 / \pi]\left[U_{1}, \ldots, U_{n}, U_{1}^{-1}, \ldots, U_{n}^{-1}\right] .
$$

Since $A_{\mathfrak{m}}$ is quasi Laurent polynomial in $n$ variables over $S_{\mathfrak{m}}$, we can write

$$
A_{\mathfrak{m}}=S_{\mathfrak{m}}\left[X_{1}, \ldots, X_{n}, f_{1}^{-1}, \ldots, f_{n}^{-1}\right],
$$

where $X_{1}, \ldots, X_{n} \in A_{\mathfrak{m}}$ are algebraically independent over $S_{\mathfrak{m}}$ and $f_{i}=a_{i} X_{i}+b_{i}($ for $i=1, \ldots, n)$.

By Lemma 3.2, without loss of generality, we can assume that

$$
S\left[U_{1}, \ldots, U_{n}, U_{1}^{-1}, \ldots, U_{n}^{-1}\right]=S\left[f_{1}, \ldots, f_{n}, f_{1}^{-1}, \ldots, f_{n}^{-1}\right] \quad(\subseteq A) .
$$

Let $K$ be the quotient field of $S$. Let $C_{i}=A \cap K\left[X_{i}\right]$, for $i=1, \ldots, n$. Note the following properties of the $S$-algebra $C_{i}$ (for each $i=1, \ldots, n$ ):

(i) $\pi A \cap C_{i}=\pi C_{i}$, and hence $\pi$ remains prime in $C_{i}$.

(ii) $\left(C_{i}\right)_{\mathfrak{m}}=C_{i} \otimes_{S} S_{\mathfrak{m}}=A_{\mathfrak{m}} \cap K\left[X_{i}\right]=S_{\mathfrak{m}}\left[X_{i}\right]=S_{\mathfrak{m}}^{[1]}$. 
(iii)

$$
\frac{S}{\pi S} \hookrightarrow \frac{S_{\mathfrak{m}}}{\pi S_{\mathfrak{m}}} \hookrightarrow \frac{A_{\mathfrak{m}}}{\pi A_{\mathfrak{m}}} .
$$

Therefore, the following composite map is injective

$$
\frac{S}{\pi S} \rightarrow \frac{C_{i}}{\pi C_{i}} \hookrightarrow \frac{A}{\pi A} \hookrightarrow \frac{A_{\mathfrak{m}}}{\pi A_{\mathfrak{m}}} .
$$

Then we have

$$
\frac{S}{\pi S} \hookrightarrow \frac{C_{i}}{\pi C_{i}} \hookrightarrow \frac{A}{\pi A} .
$$

Therefore, $\pi C_{i} \cap S=\pi S$. Also,

$$
\frac{\left(C_{i}\right)_{\mathfrak{m}}}{\pi\left(C_{i}\right)_{\mathfrak{m}}} \cong \frac{S_{\mathfrak{m}}}{\pi S_{\mathfrak{m}}}\left[X_{i}\right]
$$

(iv)

hence, tr. $\operatorname{deg}_{S / \pi S} C_{i} / \pi C_{i}=1$.

$$
\begin{aligned}
C_{i}[1 / \pi] & =A[1 / \pi] \cap K\left[X_{i}\right] \\
& =S[1 / \pi]\left[U_{1}, \ldots, U_{n}, U_{1}^{-1}, \ldots, U_{n}^{-1}\right] \cap K\left[X_{i}\right] \\
& =S[1 / \pi]\left[f_{1}, \ldots, f_{n}, f_{1}^{-1}, \ldots, f_{n}^{-1}\right] \cap K\left[f_{i}\right] \\
& =S[1 / \pi]\left[f_{i}\right]=S[1 / \pi]^{[1]} .
\end{aligned}
$$

(v) Let $y \in A / \pi A\left(\hookrightarrow A_{\mathfrak{m}} / \pi A_{\mathfrak{m}}\right)$ be algebraic over $S / \pi S$. Since $S_{\mathfrak{m}} / \pi S_{\mathfrak{m}}$ is algebraically closed in $A_{\mathfrak{m}} / \pi A_{\mathfrak{m}}$, it can be seen that $y \in A / \pi A \cap S_{\mathfrak{m}} / \pi S_{\mathfrak{m}}$. But, since $A / \pi A$ is faithfully flat over $S / \pi S$, we have $A / \pi A \cap S_{\mathfrak{m}} / \pi S_{\mathfrak{m}}=S / \pi S$. Then $y \in S / \pi S$. Therefore, $S / \pi S$ is algebraically closed in $A / \pi A$, and hence in $C_{i} / \pi C_{i}$ by (3.3).

Because of these properties of $C_{i}$, by the Russell-Sathaye criterion ([3, 2.3.1]) for an algebra to be a polynomial ring in one variable, we see that $C_{i}=S^{[1]}$, for each $i=1, \ldots, n$.

Let $C_{i}=S\left[T_{i}\right]\left(=S^{[1]}\right), 1 \leq i \leq n$. Then from (ii) we have $\left(C_{i}\right)_{\mathfrak{m}}=S_{\mathfrak{m}}\left[X_{i}\right]=S_{\mathfrak{m}}\left[T_{i}\right]$. Therefore, for each $i=1, \ldots, n$, there exist $a_{i}^{\prime} \in S_{\mathfrak{m}}^{*}$ and $b_{i}^{\prime} \in S_{\mathfrak{m}}$ such that $X_{i}=a_{i}^{\prime} T_{i}+b_{i}^{\prime}$. Then $f_{i}=a_{i} X_{i}+b_{i}=$ $a_{i} a_{i}^{\prime} T_{i}+a_{i} b_{i}^{\prime}+b_{i}$. Set $c_{i}:=a_{i} a_{i}^{\prime}$ and $d_{i}:=a_{i} b_{i}^{\prime}+b_{i}$. Note that $c_{i}, d_{i} \in S_{\mathfrak{m}}$ with $c_{i} \neq 0$. And then $f_{i}=c_{i} T_{i}+d_{i}$. Since $f_{i} \in C_{i}=S\left[T_{i}\right]$, it follows that $c_{i}, d_{i} \in S$. 
Set $A^{\prime}:=S\left[T_{1}, \ldots, T_{n}, f_{1}^{-1}, \ldots, f_{n}^{-1}\right]$.

Since $f_{i} \in\left(A^{\prime}\right)^{*}, f_{i}=c_{i} T_{i}+d_{i} \Rightarrow 1=f_{i}^{-1} c_{i} T_{i}+f_{i}^{-1} d_{i}$. Then we have $\left(c_{i}, d_{i}\right) A^{\prime}=A^{\prime}$. Also, $A^{\prime}$ is a flat subalgebra of a faithfully flat $S$-algebra $A$. Therefore, $A^{\prime}$ is faithfully flat over $S$. Hence, $\left(c_{i}, d_{i}\right) S=\left(c_{i}, d_{i}\right) A^{\prime} \cap S=A^{\prime} \cap S=S$, for each $i=1, \ldots, n$. Thus, $A^{\prime}$ is a quasi Laurent polynomial algebra in $n$ variables over $S$.

Claim. $\quad A^{\prime}=A$. First observe that $A^{\prime} \subseteq A$ and, by Lemma 2.8, we have $A^{\prime}=A^{\prime}[1 / \pi] \cap A_{\mathfrak{m}}^{\prime}, A=A[1 / \pi] \cap A_{\mathfrak{m}}$. Since $S_{\mathfrak{m}}\left[X_{i}\right]=S_{\mathfrak{m}}\left[T_{i}\right]$, we obtain

$$
\begin{aligned}
A_{\mathfrak{m}}^{\prime} & =S_{\mathfrak{m}}\left[T_{1}, \ldots, T_{n}, f_{1}^{-1}, \ldots, f_{n}^{-1}\right] \\
& =S_{\mathfrak{m}}\left[X_{1}, \ldots, X_{n}, f_{1}^{-1}, \ldots, f_{n}^{-1}\right] \\
& =A_{\mathfrak{m}} .
\end{aligned}
$$

Also, since $S\left[f_{1}, \ldots, f_{n}, f_{1}^{-1}, \ldots, f_{n}^{-1}\right] \subseteq A^{\prime} \subseteq A$, we obtain

$$
\begin{aligned}
S[1 / \pi]\left[f_{1}, \ldots, f_{n}, f_{1}^{-1}, \ldots, f_{n}^{-1}\right] & \subseteq A^{\prime}[1 / \pi] \\
& \subseteq A[1 / \pi] \\
& =S[1 / \pi]\left[U_{1}, \ldots, U_{n}, U_{1}^{-1}, \ldots, U_{n}^{-1}\right] \\
& =S[1 / \pi]\left[f_{1}, \ldots, f_{n}, f_{1}^{-1}, \ldots, f_{n}^{-1}\right] \\
& \Rightarrow A^{\prime}[1 / \pi]=A[1 / \pi] .
\end{aligned}
$$

Hence, $A^{\prime}=A^{\prime}[1 / \pi] \cap A_{\mathfrak{m}}^{\prime}=A[1 / \pi] \cap A_{\mathfrak{m}}=A$.

As a particular case of Proposition 3.3, it follows that, if $S$ is a P.I.D. and $A$ is a locally quasi Laurent polynomial algebra in $n$ variables over $S$ such that $A[1 / \pi]$ is a Laurent polynomial algebra in $n$ variables over $S[1 / \pi]$ for some prime $\pi$ in $S$, then $A$ is a quasi Laurent polynomial algebra in $n$ variables over $S$.

4. Examples. Throughout this section, $S$ denotes a semi-local P.I.D. with only two maximal ideals $\mathfrak{m}_{1}=\pi_{1} S$ and $\mathfrak{m}_{2}=\pi_{2} S$. Assume that $S$ contains the field of rationals $\mathbf{Q}$. Let $K$ be the quotient field of $S$ and $k_{1}=S / \mathfrak{m}_{1}, k_{2}=S / \mathfrak{m}_{2}$.

Since $\mathfrak{m}_{1}$ and $\mathfrak{m}_{2}$ are comaximal ideals of $S$, we see that $S_{\mathfrak{m}_{1}}=$ $S\left[1 / \pi_{2}\right]$ and $S_{\mathfrak{m}_{2}}=S\left[1 / \pi_{1}\right]$. 
In this section, we give examples of locally quasi Laurent polynomial algebras in two variables over $S$ which are not quasi Laurent polynomial. Let $A$ be such an algebra. In view of Proposition 3.3, neither $A\left[1 / \pi_{2}\right]$ nor $A\left[1 / \pi_{1}\right]$ can be a Laurent polynomial algebra in two variables over the discrete valuation rings $S\left[1 / \pi_{2}\right]$ and $S\left[1 / \pi_{1}\right]$, respectively. Hence, neither of the closed fibres $A / \pi_{1} A$ and $A / \pi_{2} A$ of $A$ can be a Laurent polynomial algebra in two variables over the respective field.

In the first example (of an $S$-algebra $A$ ) that follows, both the closed fibres of $A$ are polynomial algebras in two variables over the respective fields.

Example 4.1. Let $\lambda, \mu$ be units of $S$ such that $\lambda-1, \mu+1 \in \mathfrak{m}_{1}$ and $\lambda-2, \mu-2 \in \mathfrak{m}_{2}$. Let $B=S\left[U, V_{1}\right]\left(=S^{[2]}\right)$ and $V=U+\pi_{1} \pi_{2} V_{1}$. Let $X=\pi_{1} \pi_{2} U+\lambda, Y=\pi_{1} \pi_{2} V+\mu$ and $A=B\left[X^{-1}, Y^{-1}\right]=$ $S\left[U, V_{1}, X^{-1}, Y^{-1}\right]$.

We list below some useful facts:

(i) In $k_{1}$, the images of $\lambda$ and $\mu$ are 1 and -1 , respectively. On the other hand, in $k_{2}$, both $\lambda$ and $\mu$ have the same image 2 .

(ii) Let bar denote the images in $B / \pi_{1} B$. Then note that $\bar{X}, \bar{Y} \in$ $k_{1}^{*}, B / \pi_{1} B=k_{1}\left[\bar{U}, \overline{V_{1}}\right]$ and

$$
A / \pi_{1} A=B / \pi_{1} B\left[\bar{X}^{-1}, \bar{Y}^{-1}\right]=B / \pi_{1} B=k_{1}^{[2]} .
$$

(iii) Let tilde denote the images in $B / \pi_{2} B$. Then, $\widetilde{X}, \widetilde{Y} \in k_{2}^{*}$, $B / \pi_{2} B=k_{2}\left[\widetilde{U}, \widetilde{V_{1}}\right]$ and

$$
A / \pi_{2} A=B / \pi_{2} B\left[\tilde{X}^{-1}, \tilde{Y}^{-1}\right]=B / \pi_{2} B=k_{2}^{[2]} .
$$

(iv) $S\left[U, V_{1}\right] \subseteq A \subseteq S\left[\left[U, V_{1}\right]\right]$ (since $X, Y$ are units in $S\left[\left[U, V_{1}\right]\right]$, the second inclusion is justified). By (ii), it follows that

$$
\frac{S\left[U, V_{1}\right]}{\pi_{1} S\left[U, V_{1}\right]}\left(=\frac{A}{\pi_{1} A}\right) \hookrightarrow \frac{S\left[\left[U, V_{1}\right]\right]}{\pi_{1} S\left[\left[U, V_{1}\right]\right]} .
$$

Hence, $\pi_{1} S\left[\left[U, V_{1}\right]\right] \cap A=\pi_{1} A$. Similarly, using (iii), we obtain $\pi_{2} S\left[\left[U, V_{1}\right]\right] \cap A=\pi_{2} A$.

Claim (1). A is a locally quasi LP algebra in two variables over $S$. 
Let $f_{1}=X Y^{-1}$ and $g_{1}=Y$. Note that $X, Y \in A^{*} \Rightarrow f_{1}, g_{1} \in A^{*}$. In $S\left[\left[U, V_{1}\right]\right]$ we have,

$$
\begin{aligned}
f_{1}= & \left(\pi_{1} \pi_{2} U+\lambda\right)\left(\pi_{1} \pi_{2} V+\mu\right)^{-1} \\
= & \lambda \mu^{-1}\left(1+\pi_{1} \pi_{2} \lambda^{-1} U\right)\left(1+\pi_{1} \pi_{2} \mu^{-1} V\right)^{-1} \\
\Longrightarrow \lambda^{-1} \mu f_{1}= & 1+\left(\lambda^{-1}-\mu^{-1}\right) \pi_{1} \pi_{2} U-\left(\pi_{1} \pi_{2}\right)^{2} \mu^{-1} V_{1}- \\
& \mu^{-1}\left(\lambda^{-1}-\mu^{-1}\right)\left(\pi_{1} \pi_{2}\right)^{2} U^{2}+\left(\pi_{1} \pi_{2}\right)^{3} F
\end{aligned}
$$

where $F \in S\left[\left[U, V_{1}\right]\right] \cap A$.

Since $\lambda-2, \mu-2 \in \mathfrak{m}_{2}=\pi_{2} S, \lambda-\mu \in \pi_{2} S \Rightarrow \lambda^{-1}-\mu^{-1} \in \pi_{2} S \Rightarrow$ $\lambda^{-1}-\mu^{-1}=\pi_{2} \beta$, for some $\beta \in S$. Then, note that $\pi_{1} \nmid \beta$ in $S$.

Substituting for $\lambda^{-1}-\mu^{-1}$ in (4.1), we obtain

$$
\lambda^{-1} \mu f_{1}-1=\pi_{1} \pi_{2}^{2}\left[\beta U-\pi_{1} \mu^{-1} V_{1}-\mu^{-1} \beta \pi_{1} \pi_{2} U^{2}+\pi_{1}^{2} \pi_{2} F\right] .
$$

By (4.2), it follows that $\lambda^{-1} \mu f_{1}-1 \in A \cap \pi_{1} \pi_{2}^{2} S\left[\left[U, V_{1}\right]\right]=\pi_{1} \pi_{2}^{2} A$. If we set $T_{1}:=\left(\lambda^{-1} \mu f_{1}-1\right) / \pi_{1} \pi_{2}^{2}$, then

$$
T_{1}=\beta U-\pi_{1} \mu^{-1} V_{1}-\mu^{-1} \beta \pi_{1} \pi_{2} U^{2}+\pi_{1}^{2} \pi_{2} F \in A .
$$

Now, $g_{1}=Y=\mu+\pi_{1} \pi_{2} U+\left(\pi_{1} \pi_{2}\right)^{2} V_{1}$. Set $W_{1}:=\left(g_{1}-\mu\right) / \pi_{1} \pi_{2}$. Then we have

$$
W_{1}=\frac{g_{1}-\mu}{\pi_{1} \pi_{2}}=U+\pi_{1} \pi_{2} V_{1} \in A
$$

Note that $f_{1}=\left(\lambda \mu^{-1} \pi_{1} \pi_{2}^{2}\right) T_{1}+\lambda \mu^{-1}$ and $g_{1}=\pi_{1} \pi_{2} W_{1}+\mu$.

Let $A_{1}=S\left[T_{1}, W_{1}, f_{1}^{-1}, g_{1}^{-1}\right]$. Then $A_{1} \subset A$. Note that $\pi_{2}$ is a prime element of $A_{1}$ and $A$ both. We show that $A_{1}\left[1 / \pi_{1}\right]=A\left[1 / \pi_{1}\right]$. Clearly, $A_{1}\left[1 / \pi_{1}\right] \subseteq A\left[1 / \pi_{1}\right]$ and

$$
\begin{aligned}
A_{1}\left[1 / \pi_{1}, 1 / \pi_{2}\right] & =K\left[f_{1}, g_{1}, f_{1}^{-1}, g_{1}^{-1}\right]=K\left[X, Y, X^{-1}, Y^{-1}\right] \\
& =A\left[1 / \pi_{1}, 1 / \pi_{2}\right]\left(=A \otimes_{S} K\right) .
\end{aligned}
$$

Let tilde denote the images in $A / \pi_{2} A$. By (4.3) and (4.4), $\widetilde{T_{1}}=$ $\widetilde{\beta} \widetilde{U}-\widetilde{\pi_{1}} \widetilde{\mu}^{-1} \widetilde{V_{1}}, \widetilde{W_{1}}=\widetilde{U}$ and

$$
\frac{A_{1}\left[1 / \pi_{1}\right]}{\pi_{2} A_{1}\left[1 / \pi_{1}\right]}=\frac{A_{1}}{\pi_{2} A_{1}} \cong k_{2}\left[\widetilde{T_{1}}, \widetilde{W_{1}}\right] \hookrightarrow k_{2}\left[\widetilde{U}, \widetilde{V_{1}}\right]=\frac{A}{\pi_{2} A}=\frac{A\left[1 / \pi_{1}\right]}{\pi_{2} A\left[1 / \pi_{1}\right]}
$$


Then, by Lemma $2.5, A_{1}\left[1 / \pi_{1}\right]=A\left[1 / \pi_{1}\right]$. Hence, $A\left[1 / \pi_{1}\right]$ is a quasi Laurent polynomial algebra in two variables over $S\left[1 / \pi_{1}\right]$ given by

$$
A\left[1 / \pi_{1}\right]=S\left[1 / \pi_{1}\right]\left[T_{1}, W_{1}, f_{1}^{-1}, g_{1}^{-1}\right]
$$

Now, let $f_{2}=X Y$ and $g_{2}=Y$. Clearly, $f_{2}, g_{2} \in A^{*}$ and

$$
f_{2}=\lambda \mu+(\lambda+\mu) \pi_{1} \pi_{2} U+\lambda\left(\pi_{1} \pi_{2}\right)^{2} V_{1}+\left(\pi_{1} \pi_{2}\right)^{2} U^{2}+\left(\pi_{1} \pi_{2}\right)^{3} U V_{1} .
$$

Since $\lambda-1, \mu+1 \in \mathfrak{m}_{1}=\pi_{1} S, \lambda+\mu \in \pi_{1} S \Rightarrow \lambda+\mu=\pi_{1} \delta$ for some $\delta \in S$. Note that $\pi_{2} \nmid \delta$ in $S$. Substituting for $\lambda+\mu$ in $f_{2}$, we obtain

$$
\begin{aligned}
f_{2}-\lambda \mu & =\pi_{1}^{2} \pi_{2}\left[\delta U+\lambda \pi_{2} V_{1}+\pi_{2} U^{2}+\pi_{1} \pi_{2} U V_{1}\right] \\
\Longrightarrow T_{2}:=\frac{f_{2}-\lambda \mu}{\pi_{1}^{2} \pi_{2}} & =\delta U+\lambda \pi_{2} V_{1}+\pi_{2} U^{2}+\pi_{1} \pi_{2}^{2} U V_{1} \in A .
\end{aligned}
$$

Set $W_{2}:=\left(g_{2}-\mu\right) / \pi_{1} \pi_{2}\left(=U+\pi_{1} \pi_{2} V_{1} \in A\right)$. Note that $f_{2}=$ $\pi_{1}^{2} \pi_{2} T_{2}+\lambda \mu$ and $g_{2}=\pi_{1} \pi_{2} W_{2}+\mu$.

Let $A_{2}=S\left[T_{2}, W_{2}, f_{2}^{-1}, g_{2}^{-1}\right]$. Then, $A_{2} \subset A$ and $\pi_{1}$ is a prime element of $A_{2}$ and $A$ both. $A_{2}\left[1 / \pi_{2}\right] \subseteq A\left[1 / \pi_{2}\right]$ and $A_{2} \otimes_{S} K=$ $K\left[X, Y, X^{-1}, Y^{-1}\right]=A \otimes_{S} K$. Let bar denote the images in $A / \pi_{1} A$. Then, $\overline{T_{2}}=\bar{\delta} \bar{U}+\bar{\lambda} \overline{\pi_{2}} \overline{V_{1}}+\overline{\pi_{2}} \bar{U}^{2}$ and $\overline{W_{2}}=\bar{U}$. Also, $k_{1}\left[\overline{T_{2}}, \overline{W_{2}}\right] \hookrightarrow$ $k_{1}\left[\bar{U}, \overline{V_{1}}\right]=A / \pi_{1} A$ and again, using Lemma $2.5, A_{2}\left[1 / \pi_{2}\right]=A\left[1 / \pi_{2}\right]$. Thus,

$$
A\left[1 / \pi_{2}\right]=S\left[1 / \pi_{2}\right]\left[T_{2}, W_{2}, f_{2}^{-1}, g_{2}^{-1}\right] .
$$

Equations (4.5) and (4.6) together prove claim (1).

Remark 4.2. $A_{1} \subsetneq A$. For, if equality holds, then $A / \pi_{1} A=$ $k_{1}\left[\overline{T_{1}}, \overline{W_{1}}\right]=k_{1}[\bar{\beta} \bar{U}, \bar{U}]=k_{1}[\bar{U}]$, a contradiction to the fact that $A / \pi_{1} A=k_{1}^{[2]}$. Similarly, using images in $A / \pi_{2} A$, it follows that $A_{2} \varsubsetneqq A$.

Claim (2). $A$ is not a quasi LP algebra in two variables over $S$. Suppose it is. Let $A=S\left[T, W, f^{-1}, g^{-1}\right]$. Since $B^{*}=S^{*}$ and $A=B\left[X^{-1}, Y^{-1}\right]$, where $X, Y$ are prime elements of $B$ which are algebraically independent over $S$, by Lemma 2.6, there exist integers $m, n, r, s$ satisfying $m s-n r= \pm 1$ and units $\alpha_{1}, \alpha_{2}$ in $S$ such that 
$f=\alpha_{1} X^{m} Y^{n}$ and $g=\alpha_{2} X^{r} Y^{s}$. In $S\left[\left[U, V_{1}\right]\right]$ we have

$$
\alpha_{1}^{-1} f=\lambda^{m} \mu^{n}\left[1+\left(m \lambda^{-1}+n \mu^{-1}\right) \pi_{1} \pi_{2} U+\left(\pi_{1} \pi_{2}\right)^{2}\left(n \mu^{-1} V_{1}+F_{1}\right)\right],
$$

where $F_{1} \in S\left[\left[U, V_{1}\right]\right] \cap A$ and

$$
\alpha_{2}^{-1} g=\lambda^{r} \mu^{s}\left[1+\left(r \lambda^{-1}+s \mu^{-1}\right) \pi_{1} \pi_{2} U+\left(\pi_{1} \pi_{2}\right)^{2}\left(s \mu^{-1} V_{1}+G_{1}\right)\right],
$$

where $G_{1} \in S\left[\left[U, V_{1}\right]\right] \cap A$. Set

$$
\begin{aligned}
T_{3} & :=\frac{\lambda^{-m} \mu^{-n} \alpha_{1}^{-1} f-1}{\pi_{1} \pi_{2}} \\
& =\left(m \lambda^{-1}+n \mu^{-1}\right) U+n \pi_{1} \pi_{2} \mu^{-1} V_{1}+\pi_{1} \pi_{2} F_{1}
\end{aligned}
$$

and

$$
\begin{aligned}
W_{3}: & =\frac{\lambda^{-r} \mu^{-s} \alpha_{2}^{-1} g-1}{\pi_{1} \pi_{2}} \\
& =\left(r \lambda^{-1}+s \mu^{-1}\right) U+s \pi_{1} \pi_{2} \mu^{-1} V_{1}+\pi_{1} \pi_{2} G_{1} .
\end{aligned}
$$

Then note that $T_{3} \in A \cap K[f]=A \cap K[T]=S[T]$ and $W_{3} \in S[W]$. The images of $T_{3}$ and $W_{3}$ in $A / \pi_{1} A$ are

$$
\overline{T_{3}}=\overline{\left(m \lambda^{-1}+n \mu^{-1}\right)} \bar{U} \text { and } \overline{W_{3}}=\overline{\left(r \lambda^{-1}+s \mu^{-1}\right)} \bar{U},
$$

respectively. Since these images are algebraically dependent over $k_{1}$, by Lemma 2.4 , either $\overline{T_{3}}$ or $\overline{W_{3}}$ is an element of $k_{1}$, i.e.,

$$
\pi_{1} \mid\left(m \lambda^{-1}+n \mu^{-1}\right) \text { or }\left(r \lambda^{-1}+s \mu^{-1}\right) \text { in } S .
$$

Considering the images in $A / \pi_{2} A$, it can be shown that

$$
\pi_{2} \mid\left(m \lambda^{-1}+n \mu^{-1}\right) \text { or }\left(r \lambda^{-1}+s \mu^{-1}\right) \text { in } S .
$$

Thus, $\left(m \lambda^{-1}+n \mu^{-1}\right)\left(r \lambda^{-1}+s \mu^{-1}\right)$ is an element of both $\pi_{1} S$ and $\pi_{2} S$. Using the images of $\lambda, \mu$ in $k_{1}$ and $k_{2}$, respectively, we obtain the following:

$$
(m-n)(r-s)=0 \quad \text { and } \quad(m+n)(r+s)=0 .
$$

As $m s-n r= \pm 1$, the only two possibilities are:

$$
(m-n=0 \quad \text { and } r+s=0) \quad \text { or }(r-s=0 \quad \text { and } m+n=0) .
$$

In the first case, i.e., if $m=n$ and $s=-r$, then $m s-n r=m(s-r)=$ $m(s+s)=2 m s$. But, then $m s-n r= \pm 1 \Rightarrow 2 m s= \pm 1 \Rightarrow m s= \pm 1 / 2$, 
a contradiction. A similar contradiction is obtained in the second case. This proves claim (2).

Before moving towards the next examples, we describe two methods (Propositions 4.3 and 4.4) of obtaining quasi Laurent polynomial algebras in two variables over a discrete valuation ring containing the field of rationals $\mathbf{Q}$.

Proposition 4.3. Let $R$ be a discrete valuation ring containing the field of rationals $\mathbf{Q}$. Let $\pi R$ be its unique maximal ideal, $K$ the quotient field and $k$ the residue field. Let

$$
B=\frac{R[X, Y, Z]}{\left(\pi^{l} Z-\left(X^{r}-Y^{m}\right)\right)},
$$

where $X, Y$ are algebraically independent over $R$ and $l, r, m$ are positive integers with $r, m$ relatively prime. Let $x, y$ denote the images of $X, Y$ respectively, in $B$. Let $A=B\left[x^{-1}, y^{-1}\right]$. Then $A$ is a quasi Laurent polynomial algebra in two variables over $R$.

Proof. Let $z$ denote the image of $Z$ in $B$. Note that $\pi^{l} z=x^{r}-y^{m} \Rightarrow$ $\pi^{l}\left(y^{-m} z\right)=x^{r} y^{-m}-1$. Set $W_{1}:=y^{-m} z$ and $g_{1}:=\pi^{l} W_{1}+1\left(=x^{r} y^{-m}\right)$. Then, as $x, y$ are units in $A ; W_{1}, g_{1}, g_{1}^{-1}$ are elements of $A$. Since $m, r$ are relatively prime, there exist $s, n \in \mathbf{Z}$ such that $m s-n r=1$. Then the determinant $\left|\begin{array}{l}r-m \\ s-n\end{array}\right|=1$. Set $T_{1}:=x^{s} y^{-n}$, and let $A^{\prime}=$ $R\left[T_{1}, T_{1}^{-1}, W_{1}, g_{1}^{-1}\right]$. Then $A^{\prime} \subseteq A$. Also, $x=g_{1}^{-n} T_{1}^{m}, y=g_{1}^{-s} T_{1}^{r}$ and $z=y^{m} W_{1} \Rightarrow x, y \in A^{\prime *}, z \in A^{\prime}$. Hence, $A=A^{\prime}$.

Proposition 4.4. Let $R$ be a discrete valuation ring containing the field of rationals $\mathbf{Q}$. Let $\pi R$ be its unique maximal ideal, $K$ the quotient field and $k$ the residue field. Let

$$
B=\frac{R[X, Y, Z]}{\left(\pi^{l} Z-\left(X^{r} Y^{m}-1\right)\right)},
$$

where $X, Y$ are algebraically independent over $R$ and $l, r, m$ are positive integers with $r, m$ relatively prime. Let $x, y$ denote the images of $X, Y$ respectively, in $B$. Let $A=B\left[x^{-1}, y^{-1}\right]$. Then $A$ is a quasi Laurent polynomial algebra in two variables over $R$. 
Proof. Let $z$ denote the image of $Z$ in $B$. Since $\pi^{l} z=x^{r} y^{m}-1$, if we set $W_{1}:=z$ and $g_{1}:=\pi^{l} W_{1}+1$, then because $x, y$ are units in $A, g_{1}, g_{1}^{-1} \in A$. Now since $r, m$ are relatively prime, there exist $s, n \in \mathbf{Z}$ such that $m s-n r=1$, i.e., the determinant $|\stackrel{r}{-s} \underset{-n}{-n}|=1$. Set $T_{1}:=x^{-s} y^{-n}$, and let $A^{\prime}=R\left[T_{1}, T_{1}^{-1}, W_{1}, g_{1}^{-1}\right]$. Then $A^{\prime} \subseteq A$. Also, $x=g_{1}^{-n} T_{1}^{-m}, y=g_{1}^{s} T_{1}^{r} \Rightarrow x, y \in A^{\prime *}$ and $z=W_{1} \in A^{\prime}$. Hence, $A=A^{\prime}$.

In the next example, both the closed fibres of $A$ contain exactly one transcendental unit.

Example 4.5. Let

$$
B=\frac{S[X, Y, Z]}{\left(\pi_{1} \pi_{2} Z-\pi_{1}\left(X^{2}-Y^{3}\right)-\pi_{2}(X Y-1)\right)},
$$

where $X, Y$ are algebraically independent over $S$. Let $x, y, z$ denote the images of $X, Y$ and $Z$ respectively, in $B$. Let $A=B\left[x^{-1}, y^{-1}\right]$.

First note the following:

(I) Let bar denote the images in $B / \pi_{1} B$. Then $\bar{x} \bar{y}=1, B / \pi_{1} B=$ $k_{1}\left[\bar{x}, \bar{x}^{-1}, \bar{z}\right]$ and

$$
A / \pi_{1} A=B / \pi_{1} B\left[\bar{x}^{-1}, \bar{y}^{-1}\right]=k_{1}\left[\bar{x}, \bar{x}^{-1}, \bar{z}\right]=B / \pi_{1} B .
$$

(II) Let tilde denote the images in $B / \pi_{2} B$. Then $\widetilde{x}^{2}=\widetilde{y}^{3}$ and $B / \pi_{2} B=k_{2}[\widetilde{x}, \widetilde{y}, \widetilde{z}]$. Also

$$
B / \pi_{2} B \hookrightarrow A / \pi_{2} A=B / \pi_{2} B\left[\widetilde{x}^{-1}, \widetilde{y}^{-1}\right]=k_{2}\left[\theta, \theta^{-1}, \widetilde{z}\right],
$$

where $\theta=\widetilde{x} \widetilde{y}^{-1}$. Note that $\theta^{3}=\widetilde{x}$ and $\theta^{2}=\widetilde{y}$. $S$.

Claim (1). A is a locally quasi LP algebra in two variables over

Note that $S\left[1 / \pi_{1}\right][X, Y, Z]=S\left[1 / \pi_{1}\right]\left[X, Y, Z_{1}\right]$, where $Z_{1}=\pi_{1} Z-$ $(X Y-1)$. Therefore,

$$
B\left[1 / \pi_{1}\right]=\frac{S\left[1 / \pi_{1}\right]\left[X, Y, Z_{1}\right]}{\left(\pi_{2} Z_{1}-\pi_{1}\left(X^{2}-Y^{3}\right)\right)} .
$$

Then, $A\left[1 / \pi_{1}\right]=B\left[1 / \pi_{1}\right]\left[x^{-1}, y^{-1}\right]$ is a quasi Laurent polynomial algebra in two variables over the D.V.R. $S\left[1 / \pi_{1}\right]$ (by Proposition 4.3). 
Now, $S\left[1 / \pi_{2}\right][X, Y, Z]=S\left[1 / \pi_{2}\right]\left[X, Y, Z_{2}\right]$, where $Z_{2}=\pi_{2} Z-\left(X^{2}-\right.$ $\left.Y^{3}\right)$. Therefore,

$$
B\left[1 / \pi_{2}\right]=\frac{S\left[1 / \pi_{2}\right]\left[X, Y, Z_{2}\right]}{\left(\pi_{1} Z_{2}-\pi_{2}(X Y-1)\right)} .
$$

Then, $A\left[1 / \pi_{2}\right]=B\left[1 / \pi_{2}\right]\left[x^{-1}, y^{-1}\right]$ is a quasi Laurent polynomial algebra in two variables over the D.V.R. $S\left[1 / \pi_{2}\right]$ (by Proposition 4.4).

Claim (2). $A$ is not a quasi LP algebra in two variables over $S$. Suppose it is. Let $A=S\left[T, W, f^{-1}, g^{-1}\right]$, where $f=a T+b$ and $g=c W+d$. Since $B^{*}=S^{*}$ and $x, y$ are prime elements of $B$ which are algebraically independent over $S$, by Lemma 2.6, there exist $m, n, r, s \in \mathbf{Z}$ with $m s-n r= \pm 1$ and $\alpha_{1}, \alpha_{2} \in S^{*}$ such that $x=\alpha_{1} f^{m} g^{n}$ and $y=\alpha_{2} f^{r} g^{s}$.

Because of the structure of $A / \pi_{1} A$ and $A / \pi_{2} A$ described in (I) and (II), we have the following four possibilities:

(i) $\pi_{1}\left|a, \pi_{1} \nmid c, \pi_{2} \nmid a, \pi_{2}\right| c$,

(ii) $\pi_{1} \nmid a, \pi_{1}\left|c, \pi_{2}\right| a, \pi_{2} \nmid c$,

(iii) $\pi_{1}\left|a, \pi_{1} \nmid c, \pi_{2}\right| a, \pi_{2} \nmid c$,

(iv) $\pi_{1} \nmid a, \pi_{1}\left|c, \pi_{2} \nmid a, \pi_{2}\right| c$.

Consider case (i). In this case, $A\left[1 / \pi_{2}\right]=S\left[1 / \pi_{2}\right]\left[T, f^{-1}, g, g^{-1}\right]$. In $A / \pi_{1} A$, the image of $f$ is $\bar{f}=\bar{b}\left(\in k_{1}^{*}\right)$ and

$$
\bar{x} \bar{y}=1 \Longrightarrow \overline{\alpha_{1}} \bar{\alpha}_{2} \bar{b}^{(m+r)} \bar{g}^{(n+s)}=1 \Longrightarrow \bar{g}^{(n+s)} \in k_{1}^{*} \text {. }
$$

Therefore, $n+s=0$.

Also, $A\left[1 / \pi_{1}\right]=S\left[1 / \pi_{1}\right]\left[f, f^{-1}, W, g^{-1}\right]$. In $A / \pi_{2} A$, the image of $g$ is $\widetilde{g}=\widetilde{d} \in k_{2}^{*}$ and

$$
\widetilde{x}^{2}=\widetilde{y}^{3} \Longrightarrow \widetilde{f}^{(2 m-3 r)} \in k_{2}^{*}
$$

Therefore, $2 m-3 r=0$, and we can write $m=3 m^{\prime}, r=2 m^{\prime}$ for some integer $m^{\prime}$, so that $m s-n r=m s+s r=s(m+r)=5 s m^{\prime}$. But, then $m s-n r= \pm 1 \Rightarrow 5 \mathrm{sm}^{\prime}= \pm 1$, a contradiction. In case (ii), also, we obtain a contradiction of the same type. 
Now consider case (iv). In this case,

$$
\begin{aligned}
A\left[1 / \pi_{2}\right] & =S\left[1 / \pi_{2}\right]\left[f, f^{-1}, W, g^{-1}\right], \\
A / \pi_{1} A & =k_{1}\left[\bar{f}, \bar{f}^{-1}, \bar{W}\right]
\end{aligned}
$$

and

$$
\bar{x} \bar{y}=1 \Longrightarrow \bar{f}^{(m+r)} \in k_{1}^{*} \Longrightarrow m+r=0 .
$$

Also, in this case, we have

$$
\begin{aligned}
A\left[1 / \pi_{1}\right] & =S\left[1 / \pi_{1}\right]\left[f, f^{-1}, W, g^{-1}\right], \\
A / \pi_{2} A & =k_{2}\left[\widetilde{f}, \tilde{f}^{-1}, \widetilde{W}\right]
\end{aligned}
$$

and

$$
\widetilde{x}^{2}=\widetilde{y}^{3} \Longrightarrow \widetilde{f}^{(2 m-3 r)} \in k_{2}^{*} \Longrightarrow 2 m-3 r=0 .
$$

Solving $m+r=0$ and $2 m-3 r=0$ simultaneously, we obtain $m=r=0$. But, then $m s-n r=0$, a contradiction. A similar contradiction is obtained in case (iii). This proves Claim (2).

In the following example, one closed fibre of $A$ is a polynomial algebra in two variables whereas the other contains exactly one transcendental unit.

Example 4.6. Let $C=S[U, V]=S^{[2]}$. Let $X=\pi_{2} U+1, Y=\pi_{2}^{2} V+1$. Let $p, q(\geq 2)$ be relatively prime integers, and let

$$
B=\frac{S[U, V, Z]}{\left(\pi_{1} Z-\left\{\left(\pi_{2} U+1\right)^{q}-\left(\pi_{2}^{2} V+1\right)^{p}\right\}\right)} .
$$

Let $x, y, z$ denote the images of $X, Y$ and $Z$ respectively, in B. Let $A=B\left[x^{-1}, y^{-1}\right]$.

First note the following:

(I) $B^{*}=S^{*}$ and $x, y$ are prime elements of $B$ which are algebraically independent over $S$.

(II) $B\left[1 / \pi_{1}\right]=S\left[1 / \pi_{1}\right][U, V]$, and hence

$$
A\left[1 / \pi_{1}\right]=S\left[1 / \pi_{1}\right]\left[U, V, X^{-1}, Y^{-1}\right] .
$$


Therefore, $A\left[1 / \pi_{1}\right]$ is a quasi Laurent polynomial algebra in two variables over $S\left[1 / \pi_{1}\right]$. And

$$
A / \pi_{2} A=\frac{A\left[1 / \pi_{1}\right]}{\pi_{2} A\left[1 / \pi_{1}\right]}=k_{2}^{[2]} .
$$

(III) $S\left[1 / \pi_{2}\right][U, V]=S\left[1 / \pi_{2}\right][X, Y]$. Then we have

$$
B\left[1 / \pi_{2}\right]=\frac{S\left[1 / \pi_{2}\right][X, Y, Z]}{\left(\pi_{1} Z-\left(X^{q}-Y^{p}\right)\right)} .
$$

Therefore, $A\left[1 / \pi_{2}\right]=B\left[1 / \pi_{2}\right]\left[x^{-1}, y^{-1}\right]$ is a quasi Laurent polynomial algebra in two variables over $S\left[1 / \pi_{2}\right]$, by Proposition 4.3 .

Let bar denote the images in $A / \pi_{1} A$. Then

$$
\begin{aligned}
A / \pi_{1} A & =\frac{A\left[1 / \pi_{2}\right]}{\pi_{1} A\left[1 / \pi_{2}\right]}=k_{1}\left[\bar{x}, \bar{y}, \bar{z}, \bar{x}^{-1}, \bar{y}^{-1}\right] \\
& =k_{1}\left[\theta, \theta^{-1}, \bar{z}\right]\left(=k_{1}\left[\theta, \theta^{-1}\right]^{[1]}\right),
\end{aligned}
$$

where $\theta=\bar{x}^{p^{\prime}} \bar{y}^{\left(-q^{\prime}\right)}$ and $p^{\prime}, q^{\prime}$ are integers such that $p p^{\prime}-q q^{\prime}=$ 1. Note that $\bar{x}=\theta^{p}$ and $\bar{y}=\theta^{q}$.

(IV) Let $R=S\left[1 / \pi_{1}\right]$. Since $A\left[1 / \pi_{1}\right]=R\left[U, V, X^{-1}, Y^{-1}\right]$ and $X, Y$ are units in $R[[U, V]]$, we have $R[U, V] \subseteq A\left[1 / \pi_{1}\right] \subseteq R[[U, V]]$. Hence, $A\left[1 / \pi_{1}\right] \cap \pi_{2} R[[U, V]]=\pi_{2} A\left[1 / \pi_{1}\right]$.

From (II) and (III), $A$ is a locally quasi Laurent polynomial algebra in two variables over $S$.

Claim. $A$ is not a quasi LP algebra in two variables over $S$. Suppose it is. Let $A=S\left[T, W, f^{-1}, g^{-1}\right]$ where $f=a T+b$ and $g=c W+d$. In view of (I), by Lemma 2.6, there exist $m, n, r, s \in \mathbf{Z}$ with $m s-n r= \pm 1$ and $\alpha_{1}, \alpha_{2} \in S^{*}$ such that $f=\alpha_{1} x^{m} y^{n}, g=\alpha_{2} x^{r} y^{s}$. Since $A / \pi_{2} A=k_{2}^{[2]}, \pi_{2} \mid a$ and $\pi_{2} \mid c$.

In $R[[U, V]]$, we have

$$
\begin{aligned}
\alpha_{1}^{-1} f & =x^{m} y^{n}=\left(\pi_{2} U+1\right)^{m}\left(\pi_{2}^{2} V+1\right)^{n} \\
& =1+m \pi_{2} U+n \pi_{2}^{2} V+\pi_{2}^{2} F
\end{aligned}
$$


where $F \in R[[U, V]] \cap A\left[1 / \pi_{1}\right]$. Then

$$
\begin{aligned}
\alpha_{1}^{-1} f-1 & =m \pi_{2} U+n \pi_{2}^{2} V+\pi_{2}^{2} F \\
& \in \pi_{2} R[[U, V]] \cap A\left[1 / \pi_{1}\right]=\pi_{2} A\left[1 / \pi_{1}\right] \\
\Longrightarrow T_{1}:=\frac{\alpha_{1}^{-1} f-1}{\pi_{2}} & =m U+n \pi_{2} V+\pi_{2} F \in A\left[1 / \pi_{1}\right] .
\end{aligned}
$$

Similarly,

$$
W_{1}:=\frac{\alpha_{2}^{-1} g-1}{\pi_{2}}=r U+s \pi_{2} V+\pi_{2} G \in A\left[1 / \pi_{1}\right],
$$

where $G \in R[[U, V]] \cap A\left[1 / \pi_{1}\right]$.

Note that $T_{1} \in A\left[1 / \pi_{1}\right] \cap K[f]=A\left[1 / \pi_{1}\right] \cap K[T]=R[T]$ and $W_{1} \in R[W]$. The images $\widetilde{T_{1}}=m \widetilde{U}$ and $\widetilde{W_{1}}=r \widetilde{U}$ of $T_{1}$ and $W_{1}$, respectively, in $\left(A\left[1 / \pi_{1}\right]\right) /\left(\pi_{2} A\left[1 / \pi_{1}\right]\right)\left(=k_{2}[\widetilde{T}, \widetilde{W}]\right)$, are algebraically dependent over $k_{2}$. Therefore, by Lemma 2.4 , either $\widetilde{T_{1}}$ or $\widetilde{W}_{1} \in k_{2}$, and hence either $m=0$ or $r=0$.

As $A / \pi_{1} A=k_{1}\left[\theta, \theta^{-1}, \bar{z}\right]\left(=k_{1}\left[\theta, \theta^{-1}\right]^{[1]}\right)=k_{1}\left[\bar{T}, \bar{W}, \bar{f}^{-1}, \bar{g}^{-1}\right]$, it follows that $\pi_{1}$ divides $a$ or $c$ (but not both). Without loss of generality, we assume that $\pi_{1} \nmid a$ (and hence $\pi_{1} \mid c$ ). Then, $\bar{g} \in k_{1}^{*}$ and $A / \pi_{1} A=k_{1}\left[\bar{f}, \bar{f}^{-1}, \bar{W}\right]$. Since $g=\alpha_{2} x^{r} y^{s}$ and $\bar{y}=\theta^{q} \notin k_{1}^{*}, r$ cannot be zero. Hence, $m$ must be zero. Then

$$
\begin{aligned}
n r= \pm 1 & \Longrightarrow n= \pm 1 \Longrightarrow f=\alpha_{1} y^{ \pm 1} \\
& \Longrightarrow \bar{f}=\overline{\alpha_{1}} \bar{y}^{( \pm 1)}=\overline{\alpha_{1}} \theta^{( \pm q)},
\end{aligned}
$$

which is a contradiction, because $q \geq 2$ and

$$
\frac{A}{\pi_{1} A}=k_{1}\left[\bar{f}, \bar{f}^{-1}, \bar{W}\right]=k_{1}\left[\theta, \theta^{-1}, \bar{z}\right] .
$$




\section{REFERENCES}

1. S.M. Bhatwadekar and Neena Gupta, On locally quasi $\mathbf{A}^{*}$ algebras in codimension-one over a Noetherian normal domain, J. Pure Appl. Alg. 215 (2011), $2242-2256$.

2. - The structure of a Laurent polynomial fibration in $n$ variables, J. Algebra 353 (2012), 142-157.

3. P. Russell and A. Sathaye, On finding and cancelling variables in $k[X, Y, Z]$, J. Algebra 57 (1979), 151-166.

Department of Mathematics, College of Engineering Pune, Shivajinagar, Pune 411 005, India

Email address: aam.maths@coep.ac.in

Bhaskaracharya Pratishthana, 56/14 Erandavane, Damle Path, Off Law College Road, Pune 411 004, India

Email address: smbhatwadekar@gmail.com 